\title{
A duality for Spin Verlinde spaces and Prym theta functions
}

\author{
C. Pauly and S. Ramanan
}

April 4, 2018

\begin{abstract}
We prove canonical isomorphisms between Spin Verlinde spaces,i.e., spaces of global sections of a determinant line bundle over the moduli space of semistable $\operatorname{Spin}_{n}$-bundles over a smooth projective curve $C$, and the dual spaces of theta functions over Prym varieties of unramified double covers of $C$.
\end{abstract}

\section{Introduction}

To any smooth, projective curve $C$, one classically associates a collection of principally polarized abelian varieties: the Jacobian $J C$, parametrizing degree zero line bundles, as well as, for any unramified double cover $\tilde{C}_{\eta} \rightarrow C$, depending on a 2-torsion point $\eta \in J C[2]$, a Prym variety $P_{\eta}$. The projective geometry of the configuration $J C \cup \bigcup_{\eta} P_{\eta}$ has been much studied [M1], [VGP], [B1] and encodes e.g. the Schottky-Jung identities among theta-constants [M1].

Less classically, one can consider the moduli space $\mathcal{M}(G)$ of semistable principal $G$-bundles over the curve $C$, where $G$ is a simple and simply-connected algebraic group. For some ample line bundle $\mathcal{L}$ over $\mathcal{M}(G)$, the vector space of global sections $H^{0}(\mathcal{M}(G), \mathcal{L})$ has been identified to a space of conformal blocks arising in conformal field theory (see e.g. [S] for a survey or [LS] for a proof), which made the computation of its dimension possible. This is the celebrated Verlinde formula.

In this paper we are interested in dualities between Verlinde spaces $H^{0}(\mathcal{M}(G), \mathcal{L})$ and spaces of abelian theta functions, i.e.,sections of some multiple of a principal polarization over $J C$ or, more generally, $J C \cup \bigcup_{\eta} P_{\eta}$. Such dualities were first proved for the structure group $G=\mathrm{SL}_{2}$ and the line bundles $\mathcal{L}^{l}$ for $l=1,2,4$ [B1], [B2], [OP] or, more generally, $G=\mathrm{SL}_{n}$ and the line bundle $\mathcal{L}[\mathrm{BNR}]$, where $\mathcal{L}$ is the ample generator of the Picard group of $\mathcal{M}\left(\mathrm{SL}_{n}\right)$.

In the articles [O1] and [O2, W.M. Oxbury constructs linear maps between a Verlinde space for the complex spin group,i.e., $G=\operatorname{Spin}_{n}$, and a space of abelian theta functions over $J C \cup \bigcup_{\eta} P_{\eta}$. Our main theorem states that these linear maps are actually isomorphisms. More precisely we show

1.1. Theorem. For any curve $C$ and any integer $m \geq 1$, we have canonical isomorphisms

$$
\begin{gathered}
(1) \sum s_{\eta}^{ \pm}: \sum_{\eta \in J C[2]} H^{0}\left(P_{\eta}^{e v},(2 m+1) \Xi_{\eta}\right)_{ \pm}^{\vee} \stackrel{\sim}{\longrightarrow} H^{0}\left(\mathcal{M}^{ \pm}\left(\operatorname{Spin}_{2 m+1}\right), \Theta\left(\mathbb{C}^{2 m+1}\right)\right) \\
(2) \sum s_{\eta}^{ \pm}: \sum_{\eta \in J C[2]}\left[H^{0}\left(P_{\eta}^{e v},(2 m) \Xi_{\eta}\right)_{ \pm}^{\vee} \oplus H^{0}\left(P_{\eta}^{o d d},(2 m) \Xi_{\eta}\right)_{ \pm}^{\vee}\right] \stackrel{\sim}{\longrightarrow} H^{0}\left(\mathcal{M}^{ \pm}\left(\operatorname{Spin}_{2 m}\right), \Theta\left(\mathbb{C}^{2 m}\right)\right)
\end{gathered}
$$


We refer to section 2 and 3 for the rather technical details encoded in the notation.

We note that theorem 1.1 for the group $\operatorname{Spin}_{3}$ coincides with the above mentioned duality for the Verlinde space $H^{0}\left(\mathcal{M}\left(\mathrm{SL}_{2}\right), \mathcal{L}^{4}\right)$ under the exceptional isomorphism $\mathrm{Spin}_{3}=\mathrm{SL}_{2}$. In this case theorem 1.1 was proved in $\mathbb{R} 2$ for all curves and in $[\mathrm{OP}$ for the generic curve.

The proof of the theorem essentially goes as follows: first, we construct maps from products of Prym varieties, called $S C H_{n}$, into the moduli space $\mathcal{M}\left(\operatorname{Spin}_{n}\right)$. This is done by induction on $n$, exploiting the exceptional isomorphisms of $\operatorname{Spin}_{n}$ with algebraic groups for small values of $n$. For example, $\mathrm{SCH}_{3}$ is isomorphic to the union $J C \cup \bigcup_{\eta} P_{\eta}$. Next, we observe that the divisor in the product $\mathcal{M}\left(\operatorname{Spin}_{n}\right) \times\left(J C \cup \bigcup_{\eta} P_{\eta}\right)$, which induces the pairing of the theorem, decomposes when restricted to the variety $S C H_{n}$. Hence we obtain a factorization of the linear map of theorem 1.1. As our auxiliary variety $S C H_{n}$ is constructed from abelian varieties, we can use theorems on multiplication of theta functions (proposition 4.1 and 4.2) and dualities for second order theta functions (Prym-Wirtinger duality, proposition 5.1) in order to show, again inductively, that all maps of the factorization are injective. By the Verlinde formula, this will be enough to deduce the theorem.

It is legitimate to ask whether the isomorphism of theorem 1.1 is a particular case of more general duality. Indeed, in the case $G=\mathrm{SL}_{n}$, one constructs ([B3] section 8 and [DT]), via canonical theta divisors, pairings among $\mathrm{SL}_{n}$ (and $\mathrm{GL}_{n}$ )-Verlinde spaces, the so-called "strange duality" or "rank-level duality". Some evidence (see [O2] remark 3.4 and [OW]) suggests that this "strange duality" phenomenon should also occur for $\operatorname{Spin}_{n}$-bundles.

As we have remarked above, the duality was proved in $\mathbb{R} 2 \pi$ in the case of $\mathrm{SL}_{2}$ and the proof was later generalized by the first author to the case of Spin groups. In order to minimize the number of papers, we decided to publish our results together.

It is a pleasure to thank W.M. Oxbury for many useful discussions.

\section{Notation and preliminaries}

\subsection{Prym varieties}

Given a nonzero $\zeta \in J C[2]$, the group of 2-torsion points of the Jacobian $J C$, we consider its associated two-sheeted étale cover $\pi_{\zeta}: \tilde{C}_{\zeta} \rightarrow C$ and the norm map $\mathrm{Nm}: \operatorname{Pic}\left(\tilde{C}_{\zeta}\right) \rightarrow \operatorname{Pic}(C)$. Mumford MI] introduced the following (isomorphic) subvarieties of $\operatorname{Pic}\left(\tilde{C}_{\zeta}\right)$, called Prym varieties

$$
\mathrm{Nm}_{\zeta}^{-1}(\mathcal{O})=P_{\zeta}^{0} \cup P_{\zeta}^{\prime}, \quad \mathrm{Nm}_{\zeta}^{-1}(\omega)=P_{\zeta}^{e v} \cup P_{\zeta}^{\text {odd }}
$$

where $P_{\zeta}^{0}$ is the connected component containing the origin $\mathcal{O} \in J C$ and $P_{\zeta}^{\prime}$ is the other component. The line bundle $\omega$ is the canonical line bundle on $C$. For a description of $\mathrm{Nm}_{\zeta}^{-1}(\omega)$, see section 2.3.1 Example a). The Galois involution $\sigma_{\zeta}$ of $\tilde{C}_{\zeta}$ acts by pull-back on the four Prym varieties. We shall also denote the corresponding involutions by $\sigma_{\zeta}$. On $P_{\zeta}^{0} \cup P_{\zeta}^{\prime}$, we have $\sigma_{\zeta}(L)=L^{-1}$ and on $P_{\zeta}^{e v} \cup P_{\zeta}^{o d d}$, we have $\sigma_{\zeta}(\xi)=\omega \xi^{-1}$. In this paper we will also use

$$
P_{\zeta}^{\zeta}=\text { one connected component of } \mathrm{Nm}^{-1}(\zeta) \text {. }
$$

In order to have a consistent notation, we put $P_{0}^{0}=J C$ and $P_{0}^{\prime}=\emptyset, P_{0}^{e v}=\operatorname{Pic}^{g-1}(C)$ and $P_{0}^{\text {odd }}=\emptyset$. On the finite group $J C[2]$ we have the skew-symmetric Weil pairing which we denote by $\langle\eta, \zeta\rangle \in\{ \pm 1\}$. The group of 2-torsion points $P_{\zeta}^{0}[2]$ of the Prym $P_{\zeta}^{0}$ is isomorphic to the annihilator (w.r.t. the Weil pairing) of $\zeta \in J C[2]$,i.e.,

$$
P_{\zeta}^{0}[2] \stackrel{\pi_{\zeta}^{*}}{\longrightarrow}\langle\zeta\rangle^{\perp} /\langle\zeta\rangle .
$$


Consider $\eta \in J C[2]$ with $\langle\eta, \zeta\rangle=1$. We also denote by $\eta \in P_{\zeta}^{0}[2]$ the corresponding element under isomorphism (2.2) and by $T_{\eta}$ the translation by $\eta$ acting on $P_{\zeta}^{0}$ as well as on the other three Prym varieties (2.1).

The Prym variety $P_{\zeta}^{e v}$ comes equipped with a naturally defined reduced Riemann theta divisor $\Xi_{\zeta}$, whose set-theoretical support equals

$$
\Xi_{\zeta}:=\left\{\xi \in P_{\zeta}^{e v} \mid h^{0}\left(\tilde{C}_{\zeta}, \xi\right)>0\right\}
$$

A translate by a theta-characteristic (resp. a 2-torsion point) of $\Xi_{\zeta}$ gives (non-canonically) a symmetric theta divisor on $P_{\zeta}^{0}$ and $P_{\zeta}^{\prime}$ (resp. $P_{\zeta}^{\text {odd }}$ ) (see also section 2.2 ), which we also denote by $\Xi_{\zeta}$.

Given $\eta \in P_{\zeta}^{0}[2]$, we define the line bundle on $P_{\zeta}$

$$
\mathcal{L}_{\zeta}^{\eta}:=\mathcal{O}_{P_{\zeta}}\left(\Xi_{\zeta}+T_{\eta}^{*} \Xi_{\zeta}\right)
$$

Here $P_{\zeta}$ stands for any of the four varieties in (2.1). We note that $\Xi_{\zeta}$ is only canonically defined on $P_{\zeta}^{e v}(2.3)$, but the line bundle $\mathcal{L}_{\zeta}^{\eta}$ over any $P_{\zeta}$ does not depend on the choice of $\Xi_{\zeta}$. Moreover the involutions $\sigma_{\zeta}$ of $P_{\zeta}$ lift canonically to a linear involution $\sigma_{\zeta}^{*}$ of $H^{0}\left(P_{\zeta}, \mathcal{L}_{\zeta}^{\eta}\right)$. The subscript + (resp. - ) will denote the + (resp. - ) eigenspace of $\sigma_{\zeta}^{*}$.

\subsection{Theta-characteristics}

In this section we recall some basic results on theta-characteristics on Prym varieties. Let $\vartheta(C)$ be the set of theta-characteristics on $C$,i.e., $\vartheta(C)=\left\{\kappa \in \operatorname{Pic}^{g-1}(C) \mid \kappa^{\otimes 2} \stackrel{\sim}{\longrightarrow} \omega\right\}$, which comes equipped with a parity map $\epsilon: \vartheta(C) \rightarrow\{ \pm 1\} ; \epsilon(\kappa)=(-1)^{h^{0}(\kappa)}$. There is a 1-to-1 correspondence between $\kappa \in \vartheta(C)$ and functions $\tilde{\kappa}: J C[2] \rightarrow\{ \pm 1\}$ which satisfy $\forall \eta, \zeta \in J C[2]$

$$
\tilde{\kappa}(\eta+\zeta)=\tilde{\kappa}(\eta) \tilde{\kappa}(\zeta)\langle\eta, \zeta\rangle \text {. }
$$

The correspondence associates to $\kappa \in \vartheta(C)$ the function $\tilde{\kappa}(\eta)=(-1)^{h^{0}(\kappa \otimes \eta)+h^{0}(\kappa)}$. The group $J C[2]$ acts on $\vartheta(C)$ by tensor product and we have $\epsilon(\eta \kappa)=\epsilon(\kappa) \tilde{\kappa}(\eta)$. We define the set $\vartheta\left(P_{\zeta}^{0}\right)$ of theta-characteristics on $P_{\zeta}^{0}$ to be the set of functions $\tilde{\kappa}: P_{\zeta}^{0}[2] \cong\langle\zeta\rangle^{\perp} /\langle\zeta\rangle \rightarrow\{ \pm 1\}$ satisfying (2.4). Then we have, using the correspondence between $\kappa$ and $\tilde{\kappa}$,

$$
\vartheta\left(P_{\zeta}^{0}\right)=\{\kappa \in \vartheta(C) \mid \tilde{\kappa}(\zeta)=1\} /\langle\zeta\rangle
$$

Note that we have an equivalence $\kappa \in \vartheta\left(P_{\zeta}^{0}\right) \Longleftrightarrow \pi_{\zeta}^{*} \kappa \in P_{\zeta}^{e v}$. Given $\kappa \in \vartheta\left(P_{\zeta}^{0}\right)$, we consider the symmetric theta divisor $T_{\kappa} \Xi_{\zeta}$ on $P_{\zeta}^{0}$. We shall write $\kappa$ instead of $\pi_{\zeta}^{*} \kappa$. Let $\iota$ be the unique isomorphism of $\sigma_{\zeta}^{*} \mathcal{O}\left(T_{\kappa} \Xi_{\zeta}\right)$ with $\mathcal{O}\left(T_{\kappa} \Xi_{\zeta}\right)$ which induces the identity at the origin, then we have

$$
\iota\left(\sigma_{\zeta}^{*} s_{\kappa}\right)=\epsilon(\kappa) s_{\kappa}
$$

where $s_{\kappa}$ is the unique global section of $\mathcal{O}\left(T_{\kappa} \Xi_{\zeta}\right)$. We have similar results for the component $P_{\zeta}^{\prime}$. We define

$$
\vartheta\left(P_{\zeta}^{\prime}\right)=\{\kappa \in \vartheta(C) \mid \tilde{\kappa}(\zeta)=-1\} /\langle\zeta\rangle=\left\{\kappa \in \vartheta(C) \mid \pi_{\zeta}^{*} \kappa \in P_{\zeta}^{o d d}\right\}
$$

We choose a 2-torsion point $\alpha \in P_{\zeta}^{\prime}[2]$, which allows us to define uniquely an isomorphism $\iota$ by the same condition at $\alpha$. Then we still have equality (2.5).

\subsection{Mumford's parity conservation theorem and Pfaffian line bundles}

We recall here Mumford's parity conservation theorem M2 which says that the parity of $h^{0}$ of vector bundles on $C$ is preserved under deformation, if there exists a non-degenerate quadratic form on the family with values in $\omega$. 


\subsubsection{Examples}

a) $\forall \xi \in P_{\zeta}^{e v} \cup P_{\zeta}^{o d d}$, the rank 2 vector bundle $E=\pi_{\zeta *} \xi$ carries a quadratic form given by the Norm homomorphism Nm $: \pi_{\zeta *} \xi \rightarrow \omega$. This explains the notation $P_{\zeta}^{e v}$ and $P_{\zeta}^{\text {odd }}$, since the two components of $\mathrm{Nm}^{-1}(\omega)$ may be distinguished by the property that $h^{0}\left(\pi_{\zeta *} \xi\right)$ is even for $\xi \in P_{\zeta}^{e v}$ and odd for $\xi \in P_{\zeta}^{o d d}$.

b) Consider $\eta, \zeta \in J C[2]$ with $\langle\eta, \zeta\rangle=1 . \forall \xi \in P_{\eta}^{e v} \cup P_{\eta}^{o d d}$ and $\forall L \in P_{\zeta}^{0} \cup P_{\zeta}^{\prime}$, the rank 4 vector bundle $\pi_{\eta *} \xi \otimes \pi_{\zeta *} L$ has a quadratic form, which is obtained by multiplying the $\omega$-valued form on $\pi_{\eta *} \xi$ and the $\mathcal{O}$-valued form on $\pi_{\zeta *} L$ (see example a).

c) Consider the rank $2 n$ vector bundle $\pi_{\zeta *} \xi \otimes F$, where $\pi_{\zeta *} \xi$ is as in a) and $F$ is an orthogonal vector bundle,i.e., equipped with an $\mathcal{O}$-valued quadratic form. Multiplying the two forms gives the required $\omega$-valued form on $\pi_{\zeta *} \xi \otimes F$.

\subsubsection{Pfaffian line bundles}

Given a family $\mathcal{E}$ of vector bundles over $C$ with an $\omega$-valued quadratic form and parametrized by an integral variety $S$, one can construct a Pfaffian line bundle $\operatorname{PF}(\mathcal{E})$ over $S$ and a Pfaffian divisor $\operatorname{div} \operatorname{PF}(\mathcal{E})$, which are square roots of the determinant line bundle $\operatorname{DET}(\mathcal{E})$ and determinant divisor $\operatorname{div} \operatorname{DET}(\mathcal{E})$,i.e.,

$$
\operatorname{DET}(\mathcal{E})=\operatorname{PF}(\mathcal{E})^{\otimes 2} \quad 2 \operatorname{divPF}(\mathcal{E})=\operatorname{divDET}(\mathcal{E})
$$

For the construction of $\operatorname{PF}(\mathcal{E})$ and $\operatorname{div} \operatorname{PF}(\mathcal{E})$ we refer to proposition 7.9 of [LS]. The support of the Cartier divisor $\operatorname{div} \operatorname{PF}(\mathcal{E})$ equals

$$
\operatorname{supp} \operatorname{divPF}(\mathcal{E})=\left\{s \in S \mid h^{0}\left(\mathcal{E}_{s}\right)>0\right\} .
$$

Thus, the Pfaffian divisor associated to the family $\pi_{\zeta *} \xi$ with $\xi \in P_{\zeta}^{e v}$ (example a) is the Riemann theta divisor $\Xi_{\zeta}$ in (2.3). The Pfaffian divisors associated to the families of example b) (resp. example c) will appear in the proof of proposition 5.1 (resp. in the construction of the Prym-Spin duality, see section 3).

\section{Prym varieties and the moduli of $\operatorname{Spin}_{n}$-bundles}

In this section we recall the definition of the linear maps of theorem 1.1 (see [01, O2 , Let $\mathrm{SC}_{n}$ be the special Clifford group of a non-degenerate quadratic form of dimension $n$ and $\mathcal{M}\left(\mathrm{SC}_{n}\right)$ be the moduli space of semistable principal $\mathrm{SC}_{n}$-bundles. The spinor norm induces a surjective morphism $\mathrm{Nm}: \mathcal{M}\left(\mathrm{SC}_{n}\right) \rightarrow \operatorname{Pic}(C)$ and we define

$$
\mathcal{M}^{+}\left(\operatorname{Spin}_{n}\right)=\mathcal{M}\left(\operatorname{Spin}_{n}\right)=\mathrm{Nm}^{-1}(\mathcal{O}), \quad \mathcal{M}^{-}\left(\operatorname{Spin}_{n}\right)=\mathrm{Nm}^{-1}(\mathcal{O}(p)),
$$

where $p$ is a fixed point on $C$. In section 6 we shall give examples of the moduli spaces $\mathcal{M}^{+}\left(\operatorname{Spin}_{n}\right)$ for low values of $n$. We denote by $V \cong \mathbb{C}^{n}$ the standard orthogonal representation of $\mathrm{SC}_{n}$ and by $S$ (if $n$ is odd) and $S^{ \pm}$(if $n$ is even) the spinor and half-spinor representations. Given a Clifford or Spin-bundle $A$, we shall denote by $A(V), A(S), A\left(S^{ \pm}\right)$the induced vector bundles.

There is a natural action of $J C[2]$ on the moduli spaces $\mathcal{M}^{ \pm}\left(\operatorname{Spin}_{n}\right)$ and we recall that there are natural Galois covers

$$
\mathcal{M}^{ \pm}\left(\operatorname{Spin}_{n}\right) \stackrel{J C[2]}{\longrightarrow} \mathcal{M}^{ \pm}\left(\mathrm{SO}_{n}\right), \quad A \rightarrow A(V) .
$$


The Galois action of $J C[2]$ on the moduli $\mathcal{M}^{ \pm}\left(\operatorname{Spin}_{n}\right)$ is given by $A \mapsto \alpha . A$ for $\alpha \in J C[2]$ with

$$
(\alpha . A)(V)=A(V) \quad(\alpha \cdot A)\left(S^{ \pm}\right)=A\left(S^{ \pm}\right) \otimes \alpha
$$

The two components $\mathcal{M}^{ \pm}\left(\mathrm{SO}_{n}\right)$ of the moduli of semistable orthogonal bundles are distinguished by the second Stiefel-Whitney class of the bundles. For some general facts on orthogonal bundles, see [R1].

To construct the linear maps of theorem 1.1, it will be enough to exhibit effective Cartier divisors in the right linear systems

$$
\mathcal{D}_{2 m+1, \eta}^{ \pm} \subset P_{\eta}^{e v} \times \mathcal{M}^{ \pm}\left(\operatorname{Spin}_{2 m+1}\right), \quad \mathcal{D}_{2 m, \eta}^{ \pm} \subset\left(P_{\eta}^{e v} \cup P_{\eta}^{\text {odd }}\right) \times \mathcal{M}^{ \pm}\left(\operatorname{Spin}_{2 m}\right) .
$$

We already observed in 2.3.1 example c) that the family $\mathcal{E}:=\left\{\pi_{\eta *} \xi \otimes A(V)\right\}$ carries an $\omega$-valued form, so we define $\mathcal{D}_{n, \eta}:=\operatorname{divPF}(\mathcal{E})$. By (2.6) we have (e.g. for $n$ odd)

$$
\operatorname{supp} \mathcal{D}_{2 m+1, \eta}^{ \pm}=\left\{(\xi, A) \in P_{\eta}^{e v} \times \mathcal{M}^{ \pm}\left(\operatorname{Spin}_{2 m+1}\right) \mid h^{0}\left(C, \pi_{\eta *} \xi \otimes A(V)\right)>0\right\} .
$$

We shall use the abreviated notation $\mathcal{D}_{\eta}$, if there is no ambiguity. We notice that the divisor $\mathcal{D}_{\eta}$ does not descend to a Cartier divisor on $P_{\eta} \times \mathcal{M}^{ \pm}\left(\mathrm{SO}_{n}\right)$. We denote by $s_{\eta}^{ \pm}$their associated linear maps. The details of this construction are worked out in $\mathrm{O2}$ s section 6 .

Given a pair of semistable orthogonal vector bundles $\left(E, E^{\prime}\right)$ in $\mathcal{M}^{ \pm}\left(\mathrm{SO}_{n}\right) \times \mathcal{M}^{+}\left(\mathrm{SO}_{n^{\prime}}\right)$ we can associate its orthogonal direct sum $E \oplus E^{\prime} \in \mathcal{M}^{ \pm}\left(\mathrm{SO}_{n+n^{\prime}}\right)$, which gives rise to a morphism $\mathcal{M}^{ \pm}\left(\mathrm{SO}_{n}\right) \times \mathcal{M}^{+}\left(\mathrm{SO}_{n^{\prime}}\right) \rightarrow \mathcal{M}^{ \pm}\left(\mathrm{SO}_{n+n^{\prime}}\right)$. In the next lemma we will show that this morphism lifts to the moduli spaces of Spin-bundles.

3.1. Lemma. For any $n, m \geq 1$, there are natural morphisms

$$
\begin{aligned}
\text { 1. } \mathcal{M}^{ \pm}\left(\operatorname{Spin}_{2 n}\right) \times \mathcal{M}^{+}\left(\operatorname{Spin}_{2 m}\right) \stackrel{\iota}{\longrightarrow} \mathcal{M}^{ \pm}\left(\operatorname{Spin}_{2 n+2 m}\right) \\
\text { 2. } \mathcal{M}^{ \pm}\left(\operatorname{Spin}_{2 n+1}\right) \times \mathcal{M}^{+}\left(\operatorname{Spin}_{2 m}\right) \stackrel{\iota}{\longrightarrow} \mathcal{M}^{ \pm}\left(\operatorname{Spin}_{2 n+2 m+1}\right)
\end{aligned}
$$

which are lifts via (3.1) of the direct sum morphisms for orthogonal vector bundles. Given a pair $\left(A, A^{\prime}\right)$ of Spin-bundles, then its sum $A+A^{\prime}:=\iota\left(A, A^{\prime}\right)$ has the following properties

1. the two associated half-spinor vector bundles are

$$
\begin{aligned}
& \left(A+A^{\prime}\right)\left(S^{+}\right)=A\left(S^{+}\right) \otimes A^{\prime}\left(S^{+}\right) \oplus A\left(S^{-}\right) \otimes A^{\prime}\left(S^{-}\right) \\
& \left(A+A^{\prime}\right)\left(S^{-}\right)=A\left(S^{+}\right) \otimes A^{\prime}\left(S^{-}\right) \oplus A\left(S^{-}\right) \otimes A^{\prime}\left(S^{+}\right)
\end{aligned}
$$

2. the associated spinor vector bundle is

$$
\left(A+A^{\prime}\right)(S)=A(S) \otimes\left(A^{\prime}\left(S^{+}\right) \oplus A^{\prime}\left(S^{-}\right)\right)
$$

Moreover the pull-back by $\iota$ of the determinant line bundle decomposes $(l=2 n$ or $2 n+1)$

$$
\iota^{*} \Theta\left(\mathbb{C}^{l+2 m}\right)=\Theta\left(\mathbb{C}^{l}\right) \otimes \Theta\left(\mathbb{C}^{2 m}\right)
$$

Proof. The natural homomorphism of algebraic groups $\mathrm{SC}_{l} \times \mathrm{Spin}_{2 m} \rightarrow \mathrm{SC}_{l+2 m}$ induces a morphism at the level of Clifford (and Spin)-bundles. By [R1] prop.4.2 and [01] lemma 1.2 semistability is preserved under this operation. Thus, taking into account that the spinor norm is preserved under the above group homomorphism and that $\mathcal{M}^{ \pm}\left(\operatorname{Spin}_{n}\right)$ is a coarse moduli space, we get the above claimed morphisms. The expressions of the associated (half-)spinor bundles are easily deduced from the definitions of the (half-)spinor representations of the Spin groups. 


\section{Multiplication of theta functions}

In this section we recall some facts on theta functions over an abelian variety. Let $(A, \Theta)$ be a principally polarized abelian variety, where $\Theta$ is a symmetric divisor representing the polarization. The subscript \pm denotes the \pm eigenspaces of $H^{0}(A, m \Theta)$ under the canonical involution of $A$. Recall that $H^{0}(A, 2 \Theta)_{+}=H^{0}(A, 2 \Theta)$. We will need the following facts on multiplication maps

4.1. Proposition. If $l \geq 2$ and $m \geq 3$, then the multiplication maps

1. $H^{0}(A, l \Theta) \otimes H^{0}(A, m \Theta) \longrightarrow H^{0}(A,(l+m) \Theta)$

2. $H^{0}(A, 2 l \Theta)_{+} \otimes H^{0}(A, m \Theta) \longrightarrow H^{0}(A,(2 l+m) \Theta)$

are surjective.

For a proof of 1, we refer e.g. to [K]. For a proof of 2, see prop. 1.4.4 [Kh].

The symmetric theta divisor $\Theta$ allows us to identify $A$ with its dual variety $\hat{A}$. Let $m$ be the isogeny

$$
\begin{aligned}
m: A \times A & \longrightarrow A \times A \\
(x, y) & \longmapsto(x+y, x-y)
\end{aligned}
$$

then it is well-known that for any $\alpha \in A[2]=\hat{A}[2]$, we have

$$
m^{*} \mathcal{O}_{A}(2 \Theta \otimes \alpha) \otimes \mathcal{O}_{A}(2 \Theta \otimes \alpha)=\mathcal{O}_{A}(4 \Theta) \otimes \mathcal{O}_{A}(4 \Theta)
$$

We take global sections and take the sum over all $\alpha \in A[2]$ to get the direct sum decomposition

$$
m^{*}: \sum_{\alpha \in A[2]} H^{0}(A, 2 \Theta \otimes \alpha) \otimes H^{0}(A, 2 \Theta \otimes \alpha) \stackrel{\sim}{\longrightarrow} H^{0}(A, 4 \Theta) \otimes H^{0}(A, 4 \Theta)
$$

Since $m$ is equivariant for the canonical involution of $A \times A$, we obtain the following decomposition into \pm eigenspaces

$$
\begin{aligned}
& \sum_{\alpha \in A[2]} H_{+}^{0} \otimes H_{+}^{0} \oplus H_{-}^{0} \otimes H_{-}^{0}=\left[H^{0}(A, 4 \Theta) \otimes H^{0}(A, 4 \Theta)\right]_{+} \\
& \sum_{\alpha \in A[2]} H_{+}^{0} \otimes H_{-}^{0} \oplus H_{-}^{0} \otimes H_{+}^{0}=\left[H^{0}(A, 4 \Theta) \otimes H^{0}(A, 4 \Theta)\right]_{-}
\end{aligned}
$$

In particular, if we restrict $m$ to the diagonal $A \hookrightarrow A \times A$ we get the direct sum decomposition (in this case we get the duplication map $m_{\mid A}=[2]: A \rightarrow A ; x \mapsto 2 x$ )

$$
[2]^{*}: \sum_{\alpha \in A[2]} H^{0}(A, 2 \Theta \otimes \alpha) \stackrel{\sim}{\longrightarrow} H^{0}(A, 8 \Theta)
$$

In the next proposition we will need a more general version, $\forall n \geq 1$

$$
[2]^{*}: \sum_{\alpha \in A[2]} H^{0}(A, n \Theta \otimes \alpha) \stackrel{\sim}{\longrightarrow} H^{0}(A, 4 n \Theta)
$$

Via the Weil pairing we can identify $A[2]$ with the group of characters $\operatorname{Hom}\left(A[2], \mathbb{C}^{*}\right) ; \alpha \mapsto$ $\langle\alpha, \cdot\rangle$. The natural action of the group $A[2]$ on $A$ and $A \times A$ lifts canonically to a linear action on the RHS spaces of (4.2), (4.5) and (4.6). Then the direct sum decompositions of (4.2), (4.5) and (4.6) are precisely the character space decompositions of this group action. 
4.2. Proposition. The following multiplication maps are surjective

1. $\sum_{\alpha \in A[2]} H^{0}(A, 2 \Theta \otimes \alpha)_{ \pm} \otimes H^{0}(A, \Theta \otimes \alpha) \longrightarrow H^{0}(A, 3 \Theta)_{ \pm}$

2. $\sum_{\alpha \in A[2]} H^{0}(A, 2 \Theta \otimes \alpha)_{+} \otimes H^{0}(A, 2 \Theta \otimes \alpha)_{ \pm} \longrightarrow H^{0}(A, 4 \Theta)_{ \pm}$

Proof. First let us prove 1. By proposition 4.1.1 $(l=4, m=8)$, the multiplication map

$$
H^{0}(A, 4 \Theta) \otimes H^{0}(A, 8 \Theta) \longrightarrow H^{0}(A, 12 \Theta)
$$

is surjective. Consider the character space decomposition (4.6) of the two spaces. Since the above tensor product is compatible with the linear action of the group $A[2]$, we get a surjective map between the two character spaces of (4.7) corresponding to the zero character,i.e.,

$$
\sum_{\alpha \in A[2]} H^{0}(A, 2 \Theta \otimes \alpha) \otimes H^{0}(A, \Theta \otimes \alpha) \longrightarrow H^{0}(A, 3 \Theta)
$$

Considering \pm eigenspaces proves assertion 1. By proposition 4.1.2 $(l=4, m=8)$, the multiplication map

$$
H^{0}(A, 8 \Theta)_{+} \otimes H^{0}(A, 8 \Theta) \longrightarrow H^{0}(A, 16 \Theta)
$$

is surjective. Considering the zero character space and \pm eigenspaces, we will prove 2 .

Finally, let us denote by Heis the Heisenberg group associated to the line bundle $\mathcal{O}(2 \Theta)$ (see [M3] or [B2] page 280),i.e. a central extension of $A[2]$ by $\mathbb{C}^{*}$

$$
0 \longrightarrow \mathbb{C}^{*} \longrightarrow \text { Heis } \longrightarrow A[2] \longrightarrow 0
$$

We recall that Heis acts linearly on $H^{0}(A, 2 \Theta)$, its unique (up to conjugation) representation of level 1.

\section{The Prym-Wirtinger duality}

Consider $\eta, \zeta \in J C[2]$ such that $\langle\eta, \zeta\rangle=1$. We recall the definition of the line bundle $\mathcal{L}_{\zeta}^{\eta}=$ $\mathcal{O}_{P_{\zeta}}\left(\Xi_{\zeta}+T_{\eta}^{*} \Xi_{\zeta}\right)=\mathcal{O}_{P_{\zeta}}\left(2 \Xi_{\zeta} \otimes \eta\right)$. In the next proposition we show that exchanging the roles of $\eta$ and $\zeta$ will establish a duality at the level of global sections of $\mathcal{L}_{\zeta}^{\eta}$. We may view this duality as an analogue for Prym varieties of the well-known Wirtinger duality (put $\eta=\zeta=0$ ) for Jacobians (see [M1] page 335). This Prym-Wirtinger duality is at the heart of the proof of theorem 1.1 (section 7), as the Prym-Spin pairing "restricts" to the Prym-Wirtinger duality for suitably chosen (products of) Prym varieties in $\mathcal{M}\left(\operatorname{Spin}_{n}\right)$.

5.1. Proposition. We have the following canonical isomorphisms for any $\eta, \zeta \in J C[2]$ such that $\langle\eta, \zeta\rangle=1$

$$
\begin{array}{ll}
H^{0}\left(P_{\eta}^{e v}, \mathcal{L}_{\eta}^{\zeta}\right)_{+}^{\vee} \cong H^{0}\left(P_{\zeta}^{0}, \mathcal{L}_{\zeta}^{\eta}\right)_{+} & H^{0}\left(P_{\eta}^{o d d}, \mathcal{L}_{\eta}^{\zeta}\right)_{+}^{\vee} \cong H^{0}\left(P_{\zeta}^{0}, \mathcal{L}_{\zeta}^{\eta}\right)_{-} \\
H^{0}\left(P_{\eta}^{e v}, \mathcal{L}_{\eta}^{\zeta}\right)_{-}^{\vee} \cong H^{0}\left(P_{\zeta}^{\prime}, \mathcal{L}_{\zeta}^{\eta}\right)_{+} & H^{0}\left(P_{\eta}^{o d d}, \mathcal{L}_{\eta}^{\zeta}\right)_{-}^{\vee} \cong H^{0}\left(P_{\zeta}^{\prime}, \mathcal{L}_{\zeta}^{\eta}\right)_{-}
\end{array}
$$


Proof. We will show that the duality between the above vector spaces is given by a reduced Cartier divisor, whose set-theoretical support equals

$$
\Delta_{\eta, \zeta}^{W i r t}:=\left\{(\xi, L) \in\left(P_{\eta}^{e v} \cup P_{\eta}^{o d d}\right) \times\left(P_{\zeta}^{0} \cup P_{\zeta}^{\prime}\right) \mid h^{0}\left(C, \pi_{\eta *} \xi \otimes \pi_{\zeta *} L\right)>0\right\} .
$$

Indeed, as shown in example b) of 2.3.1, the family $\mathcal{E}$ of rank 4 vector bundles $\pi_{\eta *} \xi \otimes \pi_{\zeta *} L$ parametrized by $\left(P_{\eta}^{e v} \cup P_{\eta}^{\text {odd }}\right) \times\left(P_{\zeta}^{0} \cup P_{\zeta}^{\prime}\right)$ is equipped with an $\omega$-valued quadratic form. Hence, by 2.3.2, we can consider its associated Pfaffian divisor $\Delta_{\eta, \zeta}^{W i r t}:=\operatorname{div} \operatorname{PF}(\mathcal{E})$. By (2.6) its support equals the set in (5.3) and an easy computation shows that $\mathcal{O}\left(\Delta_{\eta, \zeta}^{\text {Wirt }}\right)=\mathcal{L}_{\eta}^{\zeta} \otimes \mathcal{L}_{\zeta}^{\eta}$. Hence we obtain a pairing

$$
H^{0}\left(P_{\eta}^{e v} \cup P_{\eta}^{o d d}, \mathcal{L}_{\eta}^{\zeta}\right)^{\vee} \stackrel{\psi}{\longrightarrow} H^{0}\left(P_{\zeta}^{0} \cup P_{\zeta}^{\prime}, \mathcal{L}_{\zeta}^{\eta}\right) .
$$

First we will show that $\psi$ is an isomorphism. We verify that both sides of (5.4) are Heis-modules (4.8) of level 1 and that $\psi$ is Heis-equivariant. Hence, since $\psi$ is nonzero, it is an isomorphism.

To finish the proof we have to analyze how $\psi$ acts on the \pm eigenspaces of the linear involutions $\sigma_{\eta}^{*}\left(\right.$ resp. $\left.\sigma_{\zeta}^{*}\right)$. Let us restrict attention to the duality on the component $P_{\eta}^{e v} \times P_{\zeta}^{0}$. Consider the rational map, induced by the divisor $\Delta_{\eta, \zeta}^{W i r t}$

$$
\begin{aligned}
\Delta: P_{\eta}^{e v} & \longrightarrow \mathbb{P} H^{0}\left(P_{\zeta}^{0}, \mathcal{L}_{\zeta}^{\eta}\right) \\
\xi & \longmapsto \Delta(\xi):=\Delta_{\eta, \zeta}^{W i r t} \mid\{\xi\} \times P_{\zeta}^{0}
\end{aligned}
$$

We observe that, $\forall \xi \in P_{\eta}^{e v}$, the divisor $\Delta(\xi)$ is invariant under the (projective) involution $\sigma_{\zeta}^{*}$. Consider $\kappa \in \vartheta(C)$ such that $\kappa \in \vartheta\left(P_{\zeta}^{0}\right) \cap \vartheta\left(P_{\eta}^{0}\right)$,i.e., $\tilde{\kappa}(\eta)=\tilde{\kappa}(\zeta)=1$. Then it follows from the definition of $\Delta_{\eta, \zeta}^{\text {Wirt }}$ that $\Delta\left(\pi_{\eta}^{*} \kappa\right)=T_{\kappa}^{*} \Xi_{\zeta}+T_{\eta \kappa}^{*} \Xi_{\zeta}$. By (2.5) and with the notation as above, we have

$$
\iota \sigma_{\zeta}^{*}\left(s_{\kappa} \cdot s_{\eta \kappa}\right)=\epsilon(\kappa) \epsilon(\eta \kappa) s_{\kappa} \cdot s_{\eta \kappa}=\tilde{\kappa}(\eta) s_{\kappa} \cdot s_{\eta \kappa}=s_{\kappa} \cdot s_{\eta \kappa}
$$

So we get $\Delta\left(\pi_{\eta}^{*} \kappa\right) \in \mathbb{P} H^{0}\left(P_{\zeta}^{0}, \mathcal{L}_{\zeta}^{\eta}\right)_{+}$. Hence im $\Delta \subset \mathbb{P} H^{0}\left(P_{\zeta}^{0}, \mathcal{L}_{\zeta}^{\eta}\right)_{+}$. On the other hand, we

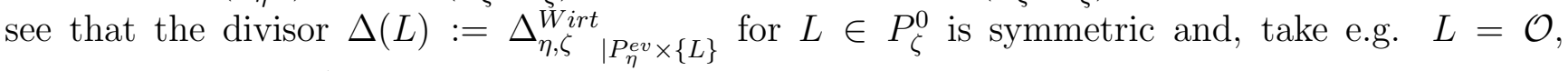
$\Delta(L) \in \mathbb{P} H^{0}\left(P_{\eta}^{e v}, \mathcal{L}_{\eta}^{\zeta}\right)_{+}$. Hence the pairing (5.4) splits as follows

$$
H^{0}\left(P_{\eta}^{e v}, \mathcal{L}_{\eta}^{\zeta}\right)_{+}^{\vee} \stackrel{\sim}{\longrightarrow} H^{0}\left(P_{\zeta}^{0}, \mathcal{L}_{\zeta}^{\eta}\right)_{+}
$$

By the same reasoning as above, one gets the other three isomorphisms as stated in the proposition. We leave the details to the reader.

\section{The Schottky variety $S C H_{n}$}

In this section we are going to define maps from products of Prym varieties into $\mathcal{M}^{ \pm}\left(\operatorname{Spin}_{n}\right)$. The disjoint union of these products will be called the Schottky variety, denoted by $S C H_{n}$. Let us start with the first cases $n \leq 6$, which will serve as a pattern to construct by induction the Schottky variety for any integer $n$.

\section{1 $n=2$}

This case has been extensively discussed in [02 section 4.2. Unlike in [02, we will define $\mathcal{M}^{+}\left(\operatorname{Spin}_{2}\right)$ to be the degree 0 component of $\mathcal{M}\left(\mathbb{C}^{*}\right)=\operatorname{Pic}(C)$, i.e., $\mathcal{M}^{+}\left(\operatorname{Spin}_{2}\right)=J C$. We also put $\mathcal{M}^{-}\left(\operatorname{Spin}_{2}\right)=\emptyset$. We define the Schottky variety by $S C H_{2}:=J C$ and the map $\phi_{2}$ is the identity $\phi_{2}: J C=\mathcal{M}^{+}\left(\operatorname{Spin}_{2}\right)$. Given $A \in \mathcal{M}^{+}\left(\operatorname{Spin}_{2}\right)$, we denote the line bundle $A\left(S^{+}\right) \in J C$ by $N$. Then we have $A\left(S^{-}\right)=N^{-1}$ and $A(V)=N^{2} \oplus N^{-2}$. 


\section{$6.2 n=3$}

In this case (see [02] section 4.3) we have the isomorphisms $\mathcal{M}^{+}\left(\operatorname{Spin}_{3}\right)=\mathcal{S} U_{C}(2, \mathcal{O})$ and $\mathcal{M}^{-}\left(\operatorname{Spin}_{3}\right)=\mathcal{S} U_{C}(2, \mathcal{O}(p))$, where we used the exceptional isomorphism $\mathrm{Spin}_{3} \cong \mathrm{SL}_{2}$ at the level of algebraic groups. Here $\mathcal{S U}_{C}(2, L)$ denotes the moduli space of semistable rank 2 vector bundles with fixed determinant equal to $L \in \operatorname{Pic}(C)$. To a semistable $\operatorname{Spin}_{3}$-bundle $A \in \mathcal{M}^{+}\left(\operatorname{Spin}_{3}\right)$ we associate two semistable vector bundles $A(S)$ (resp. $A(V)$ ) induced by the spinor (resp. orthogonal) representation. In particular the isomorphism $\mathcal{M}^{+}\left(\operatorname{Spin}_{3}\right) \stackrel{\sim}{\longrightarrow} \mathcal{S} U_{C}(2, \mathcal{O})$ is given by the map $A \mapsto A(S)$. Then the orthogonal rank 3 bundle is given by

$$
A(V)=\operatorname{End}_{0}(A(S)) \text {. }
$$

We choose a point $\tilde{p} \in \tilde{C}_{\zeta}$ lying over $p$. Taking direct image gives the following maps

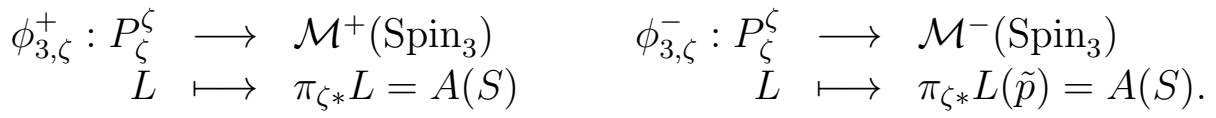

If $\zeta=0$, we define $\phi_{3,0}^{+}(L)=L \oplus L^{-1}$ and $\phi_{3,0}^{-}(L)$ is the unique stable rank 2 bundle, which fits into the exact sequence (see [B2] section 3)

$$
0 \longrightarrow L \oplus L^{-1} \longrightarrow \phi_{3,0}^{-}(L) \longrightarrow \mathbb{C}_{p} \longrightarrow 0 .
$$

6.1. Lemma. Given $M \in \operatorname{Pic}\left(\tilde{C}_{\zeta}\right)$. Then the orthogonal bundle $\operatorname{End}_{0}\left(\pi_{\zeta *} M\right)$ is an orthogonal direct sum

$$
\operatorname{End}_{0}\left(\pi_{\zeta *} M\right)=\zeta \oplus \pi_{\zeta *}\left(M^{2} \otimes \pi_{\zeta}^{*}(\mathrm{Nm} M)^{-1}\right) .
$$

Proof. Since $\pi_{\zeta}^{*} \zeta=\mathcal{O}_{\tilde{C}}$, we have an isomorphism $\zeta \otimes \pi_{\zeta *} M \stackrel{\sim}{\longrightarrow} \pi_{\zeta *} M$, which gives rise to a homomorphism $\zeta \rightarrow \operatorname{End}_{0}\left(\pi_{\zeta *} M\right)$. Since both $\zeta$ and $\operatorname{End}_{0}\left(\pi_{\zeta *} M\right)$ have degree 0 and since $\operatorname{End}_{0}\left(\pi_{\zeta *} M\right)$ is poly-stable, it follows that $\zeta$ is a subbundle of $\operatorname{End}_{0}\left(\pi_{\zeta *} M\right)$. It remains to determine its supplement bundle. There is a natural homomorphism of $\operatorname{Sym}^{2}\left(\pi_{\zeta *} M\right)$ into $\pi_{\zeta *} M^{2}$. Using the isomorphism $\operatorname{End}_{0}\left(\pi_{\zeta *} M\right) \cong \operatorname{Sym}^{2}\left(\pi_{\zeta *} M\right) \otimes(\mathrm{Nm} M)^{-1} \otimes \zeta$, we get a homomorphism of $\operatorname{End}_{0}\left(\pi_{\zeta *} M\right)$ into $\pi_{\zeta *} M^{2} \otimes(\mathrm{Nm} M)^{-1}$. A pointwise check shows that this is a surjective homomorphism and that it is supplementary to $\zeta$.

In particular, we have for $L \in P_{\zeta}^{\zeta}$

$$
\operatorname{End}_{0}\left(\pi_{\zeta *} L\right)=\zeta \oplus \pi_{\zeta *} L^{2} \quad \operatorname{End}_{0}\left(\pi_{\zeta *} L(\tilde{p})\right)=\zeta \oplus \pi_{\zeta *}\left(L^{2}(\tilde{p}-\sigma \tilde{p})\right)
$$

We define the Schottky variety and the morphism $\phi_{3}^{ \pm}$

$$
S C H_{3}:=\coprod_{\zeta \in J C[2]} P_{\zeta}^{\zeta}, \quad \phi_{3}^{ \pm}:=\coprod_{\zeta} \phi_{3, \zeta}^{ \pm}: S C H_{3} \longrightarrow \mathcal{M}^{ \pm}\left(\operatorname{Spin}_{3}\right)
$$

\section{$6.3 n=4$}

In this case (see O2 section 4.4) we have the isomorphisms $\mathcal{M}^{+}\left(\operatorname{Spin}_{4}\right)=\mathcal{S} U_{C}(2, \mathcal{O}) \times \mathcal{S} U_{C}(2, \mathcal{O})$ and $\mathcal{M}^{-}\left(\operatorname{Spin}_{4}\right)=\mathcal{S} U_{C}(2, \mathcal{O}(p)) \times \mathcal{S} U_{C}(2, \mathcal{O}(p))$, where we used the exceptional isomorphism $\operatorname{Spin}_{4}=\mathrm{SL}_{2} \times \mathrm{SL}_{2}$. The previous isomorphism is given by sending a $\mathrm{Spin}_{4}$-bundle $A$ to the pair of rank 2 bundles $\left(A\left(S^{+}\right), A\left(S^{-}\right)\right)$. Consider the maps

$$
\begin{aligned}
\phi_{4, \zeta}^{ \pm}: P_{\zeta}^{\zeta} \times P_{\zeta}^{\zeta} & \longrightarrow \mathcal{M}^{ \pm}\left(\operatorname{Spin}_{4}\right) \\
(L, M) & \longmapsto \phi_{4, \zeta}^{ \pm}(L, M):=\left(\phi_{3, \zeta}^{ \pm}(L), \phi_{3, \zeta}^{ \pm}(M)\right)
\end{aligned}
$$

where we use the previous isomorphisms. 
6.2. Lemma. Given a pair $(L, M) \in P_{\zeta}^{\zeta} \times P_{\zeta}^{\zeta}$, we consider their associated $\operatorname{Spin}_{4}$-bundles $A^{ \pm}:=$ $\phi_{4}^{ \pm}(L, M)$. Then we have

$$
A^{+}(V)=\pi_{\zeta *}(L M) \oplus \pi_{\zeta *}\left(L M^{-1}\right), \quad A^{-}(V)=\pi_{\zeta *}(L M(\tilde{p}-\sigma \tilde{p})) \oplus \pi_{\zeta *}\left(L M^{-1}\right) .
$$

Proof. We know that the orthogonal bundle $A^{ \pm}(V)$ is the tensor product $A^{ \pm}\left(S^{+}\right) \otimes A^{ \pm}\left(S^{-}\right)^{\vee}$. Let us do the computations for $A^{+}(V)$.

$$
A^{+}(V)=A^{+}\left(S^{+}\right) \otimes A^{+}\left(S^{-}\right)=\pi_{\zeta *} L \otimes \pi_{\zeta *} M=\pi_{\zeta *}\left(L \otimes \pi_{\zeta}^{*} \pi_{\zeta *} M\right)
$$

where we used the fact that $A^{+}\left(S^{-}\right)$is self-dual and the projection formula for the map $\pi_{\zeta}$. Now by example a) 2.3.1, $\pi_{\zeta}^{*} \pi_{\zeta *} M$ is a semistable orthogonal bundle over $\tilde{C}_{\zeta}$, hence this bundle splits $\pi_{\zeta}^{*} \pi_{\zeta *} M=M \oplus M^{-1}$. The computations for $A^{-}(V)$ are similar.

We define the Schottky variety and the morphism $\phi_{4}^{ \pm}$by

$$
\coprod \phi_{4, \zeta}^{ \pm}: S C H_{4}:=\coprod_{\zeta \in J C[2]} P_{\zeta}^{\zeta} \times P_{\zeta}^{\zeta} \longrightarrow \mathcal{M}^{ \pm}\left(\operatorname{Spin}_{4}\right)
$$

\section{4 $n=5$}

We define $\phi_{5, \zeta}^{ \pm}$to be the composite map (see sections 6.1, 6.2 and lemma 3.1)

$$
P_{\zeta}^{\zeta} \times J C \stackrel{\phi_{3, \zeta}^{ \pm} \times \phi_{2}}{\longrightarrow} \mathcal{M}^{ \pm}\left(\operatorname{Spin}_{3}\right) \times \mathcal{M}^{+}\left(\operatorname{Spin}_{2}\right) \stackrel{\iota}{\longrightarrow} \mathcal{M}^{ \pm}\left(\operatorname{Spin}_{5}\right)
$$

For example, given $(L, N) \in P_{\zeta}^{\zeta} \times J C$, the $\operatorname{Spin}_{5}$-bundle $A:=\phi_{5, \zeta}^{+}(L, N)$ satisfies

$$
A(S)=\left(N \oplus N^{-1}\right) \otimes \pi_{\zeta *} L \quad A(V)=\zeta \oplus N^{2} \oplus N^{-2} \oplus \pi_{\zeta *} L^{2}
$$

We remark that in this case we have an isomorphism $\mathcal{M}^{+}\left(\operatorname{Spin}_{5}\right)=\mathcal{M}^{+}\left(\operatorname{Sp}_{4}\right), A \mapsto A(S)$. The symplectic form on the bundle $A(S)$ in (6.2) is the obvious one. We define the Schottky variety by

$$
\phi_{5}^{ \pm}: S C H_{5}:=\coprod_{\zeta \in J C[2]} P_{\zeta}^{\zeta} \times J C \longrightarrow \mathcal{M}^{ \pm}\left(\operatorname{Spin}_{5}\right)
$$

\section{$6.5 n=6$}

Consider the composite map (see sections 6.1, 6.3 and lemma 3.1)

$$
\phi_{6, \zeta}^{ \pm}: P_{\zeta}^{\zeta} \times P_{\zeta}^{\zeta} \times J C \stackrel{\phi_{4, \zeta}^{ \pm} \times \phi_{2}}{\longrightarrow} \mathcal{M}^{ \pm}\left(\operatorname{Spin}_{4}\right) \times \mathcal{M}^{+}\left(\operatorname{Spin}_{2}\right) \stackrel{\iota}{\longrightarrow} \mathcal{M}^{ \pm}\left(\operatorname{Spin}_{6}\right)
$$

which associates to the triple $(L, M, N) \in P_{\zeta}^{\zeta} \times P_{\zeta}^{\zeta} \times J C$ the $\operatorname{Spin}_{6}$-bundle $A$, which verifies

$$
\begin{gathered}
A(V)=N^{2} \oplus N^{-2} \oplus \pi_{\zeta *} L M \oplus \pi_{\zeta *} L M^{-1} \\
A\left(S^{+}\right)=\left(N \otimes \pi_{\zeta *} M\right) \oplus\left(N^{-1} \otimes \pi_{\zeta *} L\right) \quad A\left(S^{-}\right)=\left(N \otimes \pi_{\zeta *} L\right) \oplus\left(N^{-1} \otimes \pi_{\zeta *} M\right)
\end{gathered}
$$

We define the Schottky variety by

$$
\phi_{6}^{ \pm}: S C H_{6}:=\coprod_{\zeta \in J C[2]} P_{\zeta}^{\zeta} \times P_{\zeta}^{\zeta} \times J C \longrightarrow \mathcal{M}^{ \pm}\left(\operatorname{Spin}_{6}\right)
$$




\section{6 the general case}

Now we will define by induction the Schottky variety $S C H_{n}$ for any integer. We put for $n \geq 3$

$$
S C H_{n+4, \zeta}:=P_{\zeta}^{\zeta} \times P_{\zeta}^{\zeta} \times S C H_{n} \quad S C H_{n+4}:=\coprod_{\zeta \in J C[2]} S C H_{n+4, \zeta}
$$

and define on each component $S C H_{n+4, \zeta}$ the morphism $\phi_{n+4, \zeta}^{ \pm}$as the composite map (see lemma 3.1)

$$
S C H_{n+4, \zeta} \stackrel{\phi_{4, \zeta}^{+} \times \phi_{n}^{ \pm}}{\longrightarrow} \mathcal{M}^{+}\left(\operatorname{Spin}_{4}\right) \times \mathcal{M}^{ \pm}\left(\operatorname{Spin}_{n}\right) \stackrel{\iota}{\longrightarrow} \mathcal{M}^{ \pm}\left(\operatorname{Spin}_{n+4}\right)
$$

Note that for $n \geq 5$ the image of $S C H_{n}$ is contained in the semistable boundary of $\mathcal{M}^{ \pm}\left(\operatorname{Spin}_{n}\right)$.

\section{$7 \quad$ Proof of theorem 1.1}

As in the previous section, we first will prove the cases $n \leq 6$ separately and then proceed by induction to prove the duality for any integer $n$.

\section{$7.1 \quad n=2$}

For the details see [02 section 4.2. In this case $\mathcal{M}^{+}\left(\operatorname{Spin}_{2}\right)=J C$ and $\Theta\left(\mathbb{C}^{2}\right)=\mathcal{O}_{J C}(8 \Theta)$. The morphism (1.2) decomposes as follows: by the Prym-Wirtinger duality (5.1) with $\zeta=0$, we have

$$
\sum_{\eta \in J C[2]} H^{0}\left(P_{\eta}^{e v}, 2 \Xi_{\eta}\right)^{\vee} \oplus H^{0}\left(P_{\eta}^{o d d}, 2 \Xi_{\eta}\right)^{\vee}=\sum_{\eta \in J C[2]} H^{0}(J C, 2 \Theta \otimes \eta)_{+} \oplus H^{0}(J C, 2 \Theta \otimes \eta)_{-},
$$

which by 4.5$)$ equals $H^{0}(J C, 8 \Theta)$.

\section{$7.2 n=3$}

First we shall consider the duality on $\mathcal{M}^{+}\left(\operatorname{Spin}_{3}\right)$. By the first equality in (6.1), we have $\forall L \in$ $P_{\zeta}^{\zeta}, \forall \xi \in P_{\eta}^{e v}$

$$
h^{0}\left(C, \operatorname{End}_{0}\left(\pi_{\zeta *} L\right) \otimes \pi_{\eta *} \xi\right)=h^{0}\left(\tilde{C}_{\eta}, \xi \otimes \pi_{\eta}^{*} \zeta\right)+h^{0}\left(C, \pi_{\zeta *} L^{2} \otimes \pi_{\eta *} \xi\right)
$$

Hence we claim that the pull-back of the divisor $\mathcal{D}_{\eta}^{+}(3.3)$ by the morphism

$$
\text { id } \times \phi_{3, \zeta}^{+}: P_{\eta}^{e v} \times P_{\zeta}^{\zeta} \longrightarrow P_{\eta}^{e v} \times \mathcal{M}^{+}\left(\operatorname{Spin}_{3}\right)
$$

splits into two divisors

$$
\left(\mathrm{id} \times \phi_{3, \zeta}^{+}\right)^{*}\left(\mathcal{D}_{\eta}^{+}\right)=p_{1}^{*}\left(T_{\zeta}^{*} \Xi_{\eta}\right)+(\mathrm{id} \times[2])^{*}\left(\Delta_{\eta, \zeta}^{W i r t}\right)
$$

where $p_{1}$ is the projection onto the first factor $P_{\eta}^{e v},[2]: P_{\zeta}^{\zeta} \rightarrow P_{\zeta}^{0}$ is the duplication map $L \mapsto L^{2}$, and $\Delta_{\eta, \zeta}^{\text {Wirt }}$ is the divisor defined in (5.3). Indeed, by (7.1) the decompositon (7.2) follows settheoretically and since $\left(\mathrm{id} \times \phi_{3, \zeta}^{+}\right)^{*}\left(\mathcal{D}_{\eta}^{+}\right) \in\left|3 \Xi_{\eta} \otimes 8 \Xi_{\zeta}\right|$, the equality (7.2) also holds schemetheoretically. The next lemma is an immediate consequence of this decomposition.

7.1. Lemma. For any $\eta, \zeta$ satisfying $\langle\eta, \zeta\rangle=1$, the linear map $s_{\eta}^{+}$composed with the pull-back induced by $\phi_{3, \zeta}^{+}$

$$
H^{0}\left(P_{\eta}^{e v}, 3 \Xi_{\eta}\right)_{+}^{\vee} \stackrel{s_{\eta}^{+}}{\longrightarrow} H^{0}\left(\mathcal{M}^{+}\left(\operatorname{Spin}_{3}\right), \Theta\left(\mathbb{C}^{3}\right)\right) \stackrel{\phi_{3, \zeta}^{+*}}{\longrightarrow} H^{0}\left(P_{\zeta}^{\zeta}, 8 \Xi_{\zeta}\right)_{+}
$$


factorizes as follows

$$
H^{0}\left(P_{\eta}^{e v}, 3 \Xi_{\eta}\right)_{+}^{\vee} \stackrel{+T_{\zeta}^{*} \Xi_{\eta}}{\longrightarrow} H^{0}\left(P_{\eta}^{e v}, \mathcal{L}_{\eta}^{\zeta}\right)_{+}^{\vee} \stackrel{\text { 5.1. }}{\longrightarrow} H^{0}\left(P_{\zeta}^{0}, \mathcal{L}_{\zeta}^{\eta}\right)_{+} \stackrel{[2]^{*}}{\longrightarrow} H^{0}\left(P_{\zeta}^{\zeta}, 8 \Xi_{\zeta}\right)_{+}
$$

where the first map is the dual of the multiplication map $D \mapsto D+T_{\zeta}^{*} \Xi_{\eta}$.

We are now in a position to prove (1.1) for $m=1$. The main idea of the proof is to show that the composite map

$$
\sum_{\eta} H^{0}\left(P_{\eta}^{e v}, 3 \Xi_{\eta}\right)_{+}^{\vee} \stackrel{\sum s_{\eta}^{+}}{\longrightarrow} H^{0}\left(\mathcal{M}^{+}\left(\operatorname{Spin}_{3}\right), \Theta\left(\mathbb{C}^{3}\right)\right) \stackrel{\phi_{3}^{+*}}{\longrightarrow} \sum_{\zeta} H^{0}\left(P_{\zeta}^{\zeta}, 8 \Xi_{\zeta}\right)_{+}
$$

is injective, which immediately implies that $\sum s_{\eta}^{+}$is an isomorphism, since the first two spaces have the same dimension ([O2] theorem 3.1).

By lemma 7.1, the linear map (7.3) factorizes as follows

$$
\begin{aligned}
\sum_{\eta \in J C[2]} H^{0}\left(P_{\eta}^{e v}, 3 \Xi_{\eta}\right)_{+}^{\vee} & \stackrel{(1)}{\longrightarrow} \sum_{\eta \in J C[2]} \sum_{\zeta \in P_{\eta}^{0}[2]} H^{0}\left(P_{\eta}^{e v}, \mathcal{L}_{\eta}^{\zeta}\right)_{+}^{\vee} \\
& \stackrel{(2)}{\longrightarrow} \sum_{\zeta \in J C[2]} \sum_{\eta \in P_{\zeta}^{0}[2]} H^{0}\left(P_{\zeta}^{0}, \mathcal{L}_{\zeta}^{\eta}\right)_{+} \\
& \stackrel{(3)}{\longrightarrow} \sum_{\zeta \in J C[2]} H^{0}\left(P_{\zeta}^{\zeta}, 8 \Xi_{\zeta}\right)_{+}
\end{aligned}
$$

The arrows are as follows: (1) is the dual of the multiplication map in proposition 4.2.1, which is injective; (2) is the Prym-Wirtinger duality (5.1), which is an isomorphism and (3) is the isomorphism induced by the duplication map (4.5). Finally we observe that we can invert the indices of summation in (2), since both sets of indices are in 1-to-1 correspondence with the set of isotropic (w.r.t the Weil form) Klein subgroups of $J C[2]$. Since the three maps (1),(2),(3) are injective, their composite map (7.3) is also injective and we are done.

Let us briefly indicate how to adapt the previous proof to the moduli $\mathcal{M}^{-}\left(\operatorname{Spin}_{3}\right)$. First, using the second equality in (6.1), we easily see that the analogue of (7.2) is

$$
\left(\mathrm{id} \times \phi_{3, \zeta}^{-}\right)^{*}\left(\mathcal{D}_{\eta}^{-}\right)=p_{1}^{*}\left(T_{\zeta}^{*} \Xi_{\eta}\right)+\left(\mathrm{id} \times\left(T_{\tilde{p}} \circ[2]\right)\right)^{*}\left(\Delta_{\eta, \zeta}^{\text {Wirt }}\right)
$$

where $T_{\tilde{p}}: P_{\zeta}^{0} \rightarrow P_{\zeta}^{\prime}$ denotes translation by $\mathcal{O}_{\tilde{C}}(\tilde{p}-\sigma \tilde{p}) \in P_{\zeta}^{\prime}$. Let us choose a square-root $\delta \in P_{\zeta}^{0}$ of $\mathcal{O}(2 \tilde{p}-2 \sigma \tilde{p}) \in P_{\zeta}^{0}$. Then we have the equality $T_{\tilde{p}}^{*} \mathcal{L}_{\zeta}^{\eta}=T_{\delta}^{*} \mathcal{L}_{\zeta}^{\eta}$ among line bundles over $P_{\zeta}^{0}$. We also recall the equality [2] $\circ T_{\epsilon}=T_{\delta} \circ[2]$, where $\epsilon \in P_{\zeta}^{0}$ is a square-root of $\delta$. Using this notation, one easily verifies that the composite map $\phi_{3, \zeta}^{-*} \circ s_{\eta}^{-}$factorizes as follows (analogue of lemma 7.1)

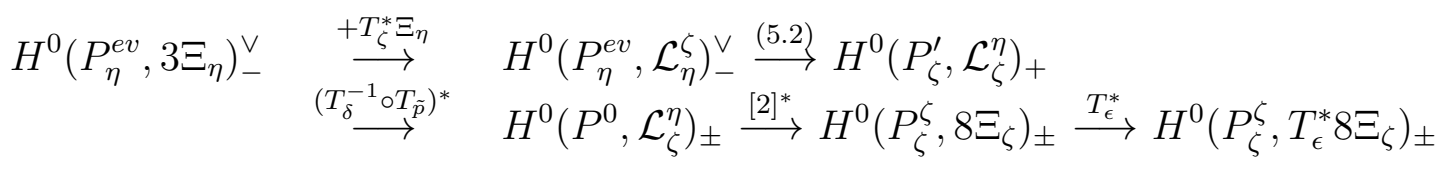

We note that the linear isomorphism $\left(T_{\delta}^{-1} \circ T_{\tilde{p}}\right)^{*}$ depends on the choice of the square-root $\delta$, which implies the indeterminacy in the sign \pm of the eigenspaces. This sign is irrelevant for the rest of the proof. The factorization (7.4) allows us to conclude as above. 


\section{$7.3 n=4$}

As in the previous section, we first consider the duality on $\mathcal{M}^{+}\left(\operatorname{Spin}_{4}\right)$. Let $(L, M) \in P_{\zeta}^{\zeta} \times P_{\zeta}^{\zeta}$ and $A=\phi_{4, \zeta}^{+}(L, M) \in \mathcal{M}^{+}\left(\operatorname{Spin}_{4}\right)$. Then we have an equality, which is a consequence of lemma 6.2: $\forall(L, M) \in P_{\zeta}^{\zeta} \times P_{\zeta}^{\zeta}, \forall \xi \in P_{\eta}^{e v} \cup P_{\eta}^{o d d}$,

$$
h^{0}\left(C, A(V) \otimes \pi_{\eta *} \xi\right)=h^{0}\left(C, \pi_{\zeta *}(L M) \otimes \pi_{\eta *} \xi\right)+h^{0}\left(C, \pi_{\zeta *}\left(L M^{-1}\right) \otimes \pi_{\eta *} \xi\right)
$$

Hence the pull-back of the divisor $\mathcal{D}_{\eta}^{+}(3.3)$ by the morphism

$$
\left(\mathrm{id} \times \phi_{4, \zeta}^{+}\right):\left(P_{\eta}^{e v} \cup P_{\eta}^{o d d}\right) \times\left(P_{\zeta}^{\zeta} \times P_{\zeta}^{\zeta}\right) \longrightarrow\left(P_{\eta}^{e v} \cup P_{\eta}^{o d d}\right) \times \mathcal{M}^{+}\left(\operatorname{Spin}_{4}\right)
$$

splits into two divisors

$$
\left(\mathrm{id} \times \phi_{4, \zeta}^{+}\right)^{*}\left(\mathcal{D}_{\eta}^{+}\right)=(\mathrm{id} \times m)^{*}\left[p_{12}^{*} \Delta_{\eta, \zeta}^{W i r t}+p_{13}^{*} \Delta_{\eta, \zeta}^{W i r t}\right]
$$

where $m$ is the isogeny $P_{\zeta}^{\zeta} \times P_{\zeta}^{\zeta} \rightarrow P_{\zeta}^{0} \times P_{\zeta}^{0}$ defined in (4.1) and $p_{i j}$ is the projection of $\left(P_{\eta}^{e v} \cup\right.$ $\left.P_{\eta}^{o d d}\right) \times P_{\zeta}^{0} \times P_{\zeta}^{0}$ onto the $i$-th and $j$-th factors. The decomposition (7.5) leads to the following factorization.

7.2. Lemma. For any $\eta, \zeta$ satisfying $\langle\eta, \zeta\rangle=1$, the linear map $s_{\eta}^{+}$composed with the pull-back induced by $\phi_{4, \zeta}^{+}$

$$
H^{0}\left(P_{\eta}^{e v}, 4 \Xi_{\eta}\right)_{+}^{\vee} \oplus H^{0}\left(P_{\eta}^{o d d}, 4 \Xi_{\eta}\right)_{+}^{\vee} \stackrel{s_{\eta}^{+}}{\longrightarrow} H^{0}\left(\mathcal{M}^{+}\left(\operatorname{Spin}_{4}\right), \Theta\left(\mathbb{C}^{4}\right)\right) \stackrel{\phi_{4, \zeta}^{+*}}{\longrightarrow} H^{0}\left(P_{\zeta}^{\zeta} \times P_{\zeta}^{\zeta}, 4 \Xi_{\zeta} \otimes 4 \Xi_{\zeta}\right)
$$

factorizes as follows

$$
\begin{array}{ll} 
& H^{0}\left(P_{\eta}^{e v}, 4 \Xi_{\eta}\right)_{+}^{\vee} \oplus H^{0}\left(P_{\eta}^{\text {odd }}, 4 \Xi_{\eta}\right)_{+}^{\vee} \\
\overrightarrow{\overrightarrow{(5.1}} & {\left[H^{0}\left(P_{\eta}^{e v}, \mathcal{L}_{\eta}^{\zeta}\right)_{+}^{\vee} \otimes H^{0}\left(P_{\eta}^{e v}, \mathcal{L}_{\eta}^{\zeta}\right)_{+}^{\vee}\right] \oplus\left[H^{0}\left(P_{\eta}^{\text {odd }}, \mathcal{L}_{\eta}^{\zeta}\right)_{+}^{\vee} \otimes H^{0}\left(P_{\eta}^{\text {odd }}, \mathcal{L}_{\eta}^{\zeta}\right)_{+}^{\vee}\right]} \\
\stackrel{m^{*}}{\longrightarrow} & {\left[H^{0}\left(P_{\zeta}^{0}, \mathcal{L}_{\zeta}^{\eta}\right)_{+} \otimes H^{0}\left(P_{\zeta}^{0}, \mathcal{L}_{\zeta}^{\eta}\right)_{+}\right] \oplus\left[H^{0}\left(P_{\zeta}^{0}, \mathcal{L}_{\zeta}^{\eta}\right)_{-} \otimes H^{0}\left(P_{\zeta}^{0}, \mathcal{L}_{\zeta}^{\eta}\right)_{-}\right]} \\
& H^{0}\left(P_{\zeta}^{\zeta} \times P_{\zeta}^{\zeta}, 4 \Xi_{\zeta} \otimes 4 \Xi_{\zeta}\right)_{+}
\end{array}
$$

where the first map is the dual of the multiplication map.

As in the $n=3$ case, it will be enough to show that the composite map

$$
\begin{aligned}
& \sum_{\eta} H^{0}\left(P_{\eta}^{e v}, 4 \Xi_{\eta}\right)_{+}^{\vee} \oplus H^{0}\left(P_{\eta}^{\text {odd }}, 4 \Xi_{\eta}\right)_{+}^{\vee} \stackrel{\sum s_{\eta}^{+}}{\longrightarrow} H^{0}\left(\mathcal{M}^{+}\left(\operatorname{Spin}_{4}\right), \Theta\left(\mathbb{C}^{4}\right)\right) \\
& \stackrel{\phi_{4}^{+*}}{\longrightarrow} \sum_{\zeta} H^{0}\left(P_{\zeta}^{\zeta} \times P_{\zeta}^{\zeta}, 4 \Xi_{\zeta} \otimes 4 \Xi_{\zeta}\right)_{+}
\end{aligned}
$$

is injective. By lemma 7.2 this linear map (7.6) factorizes as follows

$$
\begin{gathered}
\sum_{\eta \in J C[2]} H^{0}\left(P_{\eta}^{e v}, 4 \Xi_{\eta}\right)_{+}^{\vee} \oplus H^{0}\left(P_{\eta}^{o d d}, 4 \Xi_{\eta}\right)_{+}^{\vee} \\
\stackrel{(1)}{\longrightarrow} \sum_{\eta \in J C[2]} \sum_{\zeta \in P_{\eta}^{0}[2]}\left[H^{0}\left(P_{\eta}^{e v}, \mathcal{L}_{\eta}^{\zeta}\right)_{+}^{\vee} \otimes H^{0}\left(P_{\eta}^{e v}, \mathcal{L}_{\eta}^{\zeta}\right)_{+}^{\vee}\right] \oplus\left[H^{0}\left(P_{\eta}^{o d d}, \mathcal{L}_{\eta}^{\zeta}\right)_{+}^{\vee} \otimes H^{0}\left(P_{\eta}^{o d d}, \mathcal{L}_{\eta}^{\zeta}\right)_{+}^{\vee}\right] \\
\stackrel{(3)}{\longrightarrow} \quad \sum_{\zeta \in J C[2]} \sum_{\eta \in P_{\zeta}^{0}[2]}\left[H^{0}\left(P_{\zeta}^{0}, \mathcal{L}_{\zeta}^{\eta}\right)_{+} \otimes H^{0}\left(P_{\zeta}^{0}, \mathcal{L}_{\zeta}^{\eta}\right)_{+}\right] \oplus\left[H^{0}\left(P_{\zeta}^{0}, \mathcal{L}_{\zeta}^{\eta}\right)_{-} \otimes H^{0}\left(P_{\zeta}^{0}, \mathcal{L}_{\zeta}^{\eta}\right)_{-}\right] \\
\quad \sum_{\zeta \in J C[2]} H^{0}\left(P_{\zeta}^{\zeta} \times P_{\zeta}^{\zeta}, 4 \Xi_{\zeta} \otimes 4 \Xi_{\zeta}\right)_{+}
\end{gathered}
$$


Map (1) is the dual of the multiplication map in proposition 4.2.2, hence is injective. Map (2) is the Prym-Wirtinger duality (5.1), and map (3) is the isomorphism (4.3). Hence the composite map is injective.

In order to avoid repetition, we will just indicate the changes to be done to adapt the previous proof to $\mathcal{M}^{-}\left(\operatorname{Spin}_{4}\right)$. The analogue of $(7.5)$ is

$$
\left(\mathrm{id} \times \phi_{4, \zeta}^{-}\right)^{*}\left(\mathcal{D}_{\eta}^{-}\right)=\left(\mathrm{id} \times\left(T_{\tilde{p}} \times \mathrm{id}\right) \circ m\right)^{*}\left[p_{12}^{*} \Delta_{\eta, \zeta}^{W i r t}+p_{13}^{*} \Delta_{\eta, \zeta}^{W i r t}\right]
$$

where the RHS-divisor is taken in $\left(P_{\eta}^{e v} \cup P_{\eta}^{o d d}\right) \times P_{\zeta}^{\prime} \times P_{\zeta}^{0}$. This implies that the composite map $\phi_{4, \zeta}^{-*} \circ s_{\eta}^{-}$factorizes as follows (analogue of lemma 7.2)

$$
\begin{aligned}
& H^{0}\left(P_{\eta}^{e v}, 4 \Xi_{\eta}\right)_{-}^{\vee} \oplus H^{0}\left(P_{\eta}^{o d d}, 4 \Xi_{\eta}\right)_{-}^{\vee} \\
& \underset{(5.1](5.2)}{\overrightarrow{5.21}}\left[H^{0}\left(P_{\eta}^{e v}, \mathcal{L}_{\eta}^{\zeta}\right)_{-}^{\vee} \otimes H^{0}\left(P_{\eta}^{e v}, \mathcal{L}_{\eta}^{\zeta}\right)_{+}^{\vee}\right] \oplus\left[H^{0}\left(P_{\eta}^{o d d}, \mathcal{L}_{\eta}^{\zeta}\right)_{-}^{\vee} \otimes H^{0}\left(P_{\eta}^{\text {odd }}, \mathcal{L}_{\eta}^{\zeta}\right)_{+}^{\vee}\right]
\end{aligned}
$$

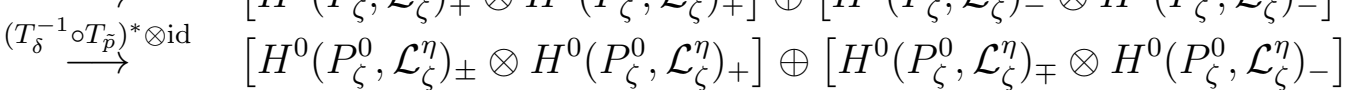

$$
\begin{aligned}
& \stackrel{m^{*}}{\longrightarrow} \quad H^{0}\left(P_{\zeta}^{\zeta} \times P_{\zeta}^{\zeta}, 4 \Xi_{\zeta} \otimes 4 \Xi_{\zeta}\right)_{ \pm} \stackrel{\left(T_{\epsilon} \times T_{\epsilon}\right)^{*}}{\longrightarrow} H^{0}\left(P_{\zeta}^{\zeta} \times P_{\zeta}^{\zeta}, T_{\epsilon}^{*} 4 \Xi_{\zeta} \otimes T_{\epsilon}^{*} 4 \Xi_{\zeta}\right)_{ \pm}
\end{aligned}
$$

where we used the equality $\left(T_{\delta} \times \mathrm{id}\right) \circ m=m \circ\left(T_{\epsilon} \times T_{\epsilon}\right)$. Now we conclude as above.

\section{$7.4 n=5$}

Since the method of the proof is the same as for $n=3$ and $n=4$, we will just indicate the main steps. Again we start with $\mathcal{M}^{+}\left(\operatorname{Spin}_{5}\right)$. As a consequence of $(6.2)$, we see that the pull-back of the divisor $\mathcal{D}_{\eta}^{+}$by the morphism id $\times \phi_{5, \zeta}^{+}$splits as follows (notation as above)

$$
\left(\mathrm{id} \times \phi_{5, \zeta}^{+}\right)^{*}\left(\mathcal{D}_{\eta}^{+}\right)=p_{1}^{*}\left(T_{\zeta}^{*} \Xi_{\eta}\right)+p_{23}^{*}\left(\left(\mathrm{id} \times[2]^{*}\right)\left(\Delta_{\eta, 0}^{W i r t}\right)\right)+p_{13}^{*}\left(\left(\mathrm{id} \times[2]^{*}\right)\left(\Delta_{\eta, \zeta}^{W i r t}\right)\right)
$$

This decomposition implies that the composite map $\phi_{5, \zeta}^{+*} \circ s_{\eta}^{+}$factorizes as follows

$$
\begin{array}{r}
H^{0}\left(P_{\eta}^{e v}, 5 \Xi_{\eta}\right)_{+}^{\vee} \stackrel{+T_{\zeta}^{*} \Xi_{\eta}}{\longrightarrow} H^{0}\left(P_{\eta}^{e v}, 2 \Xi_{\eta}\right)^{\vee} \otimes H^{0}\left(P_{\eta}^{e v}, \mathcal{L}_{\eta}^{\zeta}\right)_{+}^{\vee} \\
\stackrel{\text { 5.1. }}{\longrightarrow} H^{0}(J C, 2 \Theta \otimes \eta)_{+} \otimes H^{0}\left(P_{\zeta}^{0}, \mathcal{L}_{\zeta}^{\eta}\right)_{+} \stackrel{[2]^{*}}{\longrightarrow} H^{0}(J C, 8 \Theta)_{+} \otimes H^{0}\left(P_{\zeta}^{\zeta}, 8 \Xi_{\zeta}\right)_{+}
\end{array}
$$

As in the previous sections, the composite map $\phi_{5}^{+*} \circ\left(\sum_{\eta} s_{\eta}^{+}\right)$factorizes

$$
\begin{aligned}
\sum_{\eta \in J C[2]} H^{0}\left(P_{\eta}^{e v}, 5 \Xi_{\eta}\right)_{+}^{\vee} & \stackrel{(1)}{\longrightarrow} \sum_{\eta \in J C[2]} H^{0}\left(P_{\eta}^{e v}, 2 \Xi_{\eta}\right)^{\vee} \otimes H^{0}\left(P_{\eta}^{e v}, 3 \Xi_{\eta}\right)_{+}^{\vee} \\
& \stackrel{(2)}{\longrightarrow} \sum_{\eta \in J C[2]} \sum_{\zeta \in P_{\eta}^{0}[2]} H^{0}\left(P_{\eta}^{e v}, 2 \Xi_{\eta}\right)^{\vee} \otimes H^{0}\left(P_{\eta}^{e v}, \mathcal{L}_{\eta}^{\zeta}\right)_{+}^{\vee} \\
& \stackrel{(3)}{\longrightarrow} \sum_{\zeta \in J C[2]} \sum_{\eta \in P_{\zeta}^{0}[2]} H^{0}(J C, 2 \Theta \otimes \eta)_{+} \otimes H^{0}\left(P_{\zeta}^{0}, \mathcal{L}_{\zeta}^{\eta}\right)_{+} \\
& \sum_{\zeta \in J C[2]} H^{0}(J C, 8 \Theta)_{+} \otimes H^{0}\left(P_{\zeta}^{\zeta}, 8 \Xi_{\zeta}\right)_{+}
\end{aligned}
$$

The map (1) is the dual of the (even part) multiplication map in proposition 4.1.1 $(l=$ $2, m=3)$. The map (2) is the dual of the multiplication map in proposition 4.2.1 tensored with $H^{0}\left(P_{\eta}^{e v}, 2 \Xi_{\eta}\right)^{\vee}$. Map (3) is Prym-Wirtinger duality (5.1) (take $\zeta=0$ on the first factor), and map (4) is an injection, by (4.5). Since all four linear maps are injective, we are done.

The proof of the duality for $\mathcal{M}^{-}\left(\operatorname{Spin}_{5}\right)$ does not present any difficulty and we leave it to the reader. 


\section{$7.5 n=6$}

The duality for $\mathcal{M}^{+}\left(\operatorname{Spin}_{6}\right)$ can be proved by induction (see next section) since we know that it holds for $\mathcal{M}^{+}\left(\operatorname{Spin}_{2}\right)$. Since $\mathcal{M}^{-}\left(\operatorname{Spin}_{2}\right)=\emptyset$, we have to deal with this case separately. Again the proof is similar to the previous ones and we will omit it.

\section{6 the general case}

In this section we shall prove theorem 1.1 by induction on $n$. We will also denote by $\Theta(V)$ the pull-back by $\phi_{n}^{ \pm}$of the determinant line bundle $\Theta(V)=\Theta\left(\mathbb{C}^{n}\right)$ to the Schottky variety $S C H_{n}$. Our induction hypothesis $\mathcal{H}_{n}$ will be the following

$\mathcal{H}_{n}$ for $n$ odd:

$$
\phi_{n}^{ \pm *} \circ\left(\sum s_{\eta}^{ \pm}\right): \sum_{\eta} H^{0}\left(P_{\eta}^{e v}, n \Xi_{\eta}\right)_{ \pm}^{\vee} \longrightarrow H^{0}\left(S C H_{n}, \Theta(V)\right) \text { is injective }
$$

$\mathcal{H}_{n}$ for $n$ even:

$$
\phi_{n}^{ \pm *} \circ\left(\sum s_{\eta}^{ \pm}\right): \sum_{\eta} H^{0}\left(P_{\eta}^{e v}, n \Xi_{\eta}\right)_{ \pm}^{\vee} \oplus H^{0}\left(P_{\eta}^{o d d}, n \Xi_{\eta}\right)_{ \pm}^{\vee} \longrightarrow H^{0}\left(S C H_{n}, \Theta(V)\right) \text { is injective }
$$

Since ([02 theorem 3.1) the LHS-space and $H^{0}\left(\mathcal{M}^{ \pm}\left(\operatorname{Spin}_{n}\right), \Theta(V)\right)$ have the same dimension (see remark 3 of section 8 for $\mathcal{M}^{-}\left(\operatorname{Spin}_{n}\right)$ ), the assumption $\mathcal{H}_{n}$ implies theorem 1.1. We already proved $\mathcal{H}_{n}$ for $2 \leq n \leq 6$. Let us assume $\mathcal{H}_{n}$ and prove $\mathcal{H}_{n+4}$. First we easily verify that the pull-back of the divisor $\mathcal{D}_{n+4, \eta}^{ \pm}$under the natural map (e.g. for $n$ odd) (see lemma 3.1)

$$
\text { id } \times \iota: P_{\eta}^{e v} \times \mathcal{M}^{+}\left(\operatorname{Spin}_{4}\right) \times \mathcal{M}^{ \pm}\left(\operatorname{Spin}_{n}\right) \longrightarrow P_{\eta}^{e v} \times \mathcal{M}^{ \pm}\left(\operatorname{Spin}_{n+4}\right)
$$

splits into two divisors

$$
(\mathrm{id} \times \iota)^{*}\left(\mathcal{D}_{n+4, \eta}^{ \pm}\right)=p_{12}^{*}\left(\mathcal{D}_{4, \eta}^{+}\right)+p_{13}^{*}\left(\mathcal{D}_{n, \eta}^{ \pm}\right) .
$$

We distinguish two cases.

\subsection{1 $n$ odd}

As a consequence of the decomposition (7.7) and the proof of the $n=4$ case, the map $\phi_{n+4}^{ \pm *} \circ\left(\sum s_{\eta}^{ \pm}\right)$ factorizes as follows

$$
\begin{array}{cc} 
& \sum_{\eta \in J C[2]} H^{0}\left(P_{\eta}^{e v},(n+4) \Xi_{\eta}\right)_{ \pm}^{\vee} \\
\stackrel{(1)}{\longrightarrow} \quad \sum_{\eta \in J C[2]} H^{0}\left(P_{\eta}^{e v}, 4 \Xi_{\eta}\right)_{+}^{\vee} \otimes H^{0}\left(P_{\eta}^{e v}, n \Xi_{\eta}\right)_{ \pm}^{\vee} \\
\stackrel{(2)}{\longrightarrow} \quad \sum_{\eta \in J C[2]} \sum_{\zeta \in P_{\eta}^{0}[2]} H^{0}\left(P_{\eta}^{e v}, \mathcal{L}_{\eta}^{\zeta}\right)_{+}^{\vee} \otimes H^{0}\left(P_{\eta}^{e v}, \mathcal{L}_{\eta}^{\zeta}\right)_{+}^{\vee} \otimes H^{0}\left(P_{\eta}^{e v}, n \Xi_{\eta}\right)_{ \pm}^{\vee} \\
\stackrel{(3)}{\longrightarrow} \quad \sum_{\zeta \in J C[2]} \sum_{\eta \in P_{\zeta}^{0}[2]} H^{0}\left(P_{\zeta}^{0}, \mathcal{L}_{\zeta}^{\eta}\right)_{+} \otimes H^{0}\left(P_{\zeta}^{0}, \mathcal{L}_{\zeta}^{\eta}\right)_{+} \otimes H^{0}\left(P_{\eta}^{e v}, n \Xi_{\eta}\right)_{ \pm}^{\vee} \\
\stackrel{{ }_{(5)}^{\longrightarrow}}{\quad} \quad \sum_{\zeta \in J C[2]}\left[\sum_{\eta \in P_{\zeta}^{0}[2]} H^{0}\left(P_{\zeta}^{0}, \mathcal{L}_{\zeta}^{\eta}\right)_{+} \otimes H^{0}\left(P_{\zeta}^{0}, \mathcal{L}_{\zeta}^{\eta}\right)_{+}\right] \otimes\left[\sum_{\eta \in J C[2]} H^{0}\left(P_{\eta}^{e v}, n \Xi_{\eta}\right)_{ \pm}^{\vee}\right] \\
\sum_{\zeta \in J C[2]} H^{0}\left(P_{\zeta}^{\zeta} \times P_{\zeta}^{\zeta}, 4 \Xi_{\zeta} \otimes 4 \Xi_{\zeta}\right) \otimes H^{0}\left(S C H_{n}, \Theta(V)\right)=H^{0}\left(S C H_{n+4}, \Theta(V)\right)
\end{array}
$$


The maps are as follows: (1) is the dual of the multiplication map in proposition $4.1 .2(l=$ $2, m=n)$. If $n=2$, we use proposition 4.1.1 $(l=2, m=4)$. (2) is the dual of the (even part) multiplication map in proposition 4.2.2. (3) is the Prym-Wirtinger duality (5.1) tensored with $H^{0}\left(P_{\eta}^{e v}, n \Xi_{\eta}\right)_{ \pm}^{\vee}$. (4) is the inclusion $H^{0}\left(P_{\eta}^{e v}, n \Xi_{\eta}\right)_{ \pm}^{\vee} \hookrightarrow \sum_{\eta} H^{0}\left(P_{\eta}^{e v}, n \Xi_{\eta}\right)_{ \pm}^{\vee}$. (5) is an injection coming from the direct sum decomposition (4.3) tensored with the injective map of $\mathcal{H}_{n}$. Finally the last equality follows from the definition of $\mathrm{SCH}_{n+4}$. Since all linear maps are injective, the composite map is injective and we have proved $\mathcal{H}_{n+4}$.

\subsection{2 $n$ even}

Since this case is similar to the odd case, we just indicate the minor changes to be done on the sequence of maps $(1), \ldots,(5)$. We take into account the additional factors $\sum_{\eta} H^{0}\left(P^{\text {odd }},(n+4) \Xi_{\eta}\right)_{ \pm}^{\vee}$, for which we can write down the maps $(1), \ldots,(4)$ with $P_{\eta}^{e v}$ replaced by $P_{\eta}^{\text {odd }}$ and $H^{0}\left(P_{\zeta}^{0}, \mathcal{L}_{\zeta}^{\eta}\right)_{+}$by $H^{0}\left(P_{\zeta}^{0}, \mathcal{L}_{\zeta}^{\eta}\right)_{-}\left(\right.$see (5.1) ). Thus adding the two copies of map (5) (written for $P^{e v}$ and $P^{\text {odd }}$ ), we observe that we still have an injection coming from (4.3).

\section{Final remarks}

1. Let us briefly indicate why we used the name "Schottky variety" for the products of Prym varieties. Indeed, for $n=3$, the image of $\phi_{3}$ consists of a union of Kummer varieties of Pryms (resp. of the Jacobian) which intersect along some 4-torsion points (resp. 2-torsion points). We refer to [vGP], [D1], [P] for a proof of these intersection properties, which may also be deduced from lemma 6.1. The coordinates of the intersection points may be interpreted as the famous Schottky-Jung identities among theta-constants. Let us call the image $\phi_{3}\left(S C H_{3}\right) \subset \mathcal{S} U_{C}(2, \mathcal{O})$ the Schottky configuration, which we embed in projective space $\mathbb{P}^{2^{g}-1}=|\mathcal{L}|^{\vee}$, where $\mathcal{L}$ is the ample generator of $\operatorname{Pic}\left(\mathcal{S} U_{C}(2, \mathcal{O})\right)$. In particular, we have $\Theta\left(\mathbb{C}^{3}\right)=\mathcal{L}^{4}$. Then we can deduce from the injectivity of $(7.3)$

8.1. Corollary. If a quartic in $\mathbb{P}^{2^{g}-1}$ vanishes on the Schottky configuration, then it vanishes on the whole of $\mathcal{S} U_{C}(2, \mathcal{O})$.

This corollary was already proved in vGP corollary 2. Moreover we have been able to show that $\mathcal{S} U_{C}(2, \mathcal{O}) \subset \mathbb{P}^{2^{g}-1}$ is defined by quartic equations. One can thus recover $\mathcal{S} U_{C}(2, \mathcal{O})$ from the Schottky configuration purely geometrically. One might speculate whether, given the Schottky configuration of abelian varieties, one might reconstruct the curve $C$. This is the "small Schottky" conjecture, see D2].

2. As observed in O2 remark 1.2 and [LS section 7.10, the reduced divisors $D_{\kappa}^{(n)}$, with $\kappa \in \vartheta(C)$, whose support is given by

$$
\operatorname{supp} D_{\kappa}^{(n)}=\left\{A \in \mathcal{M}^{+}\left(\operatorname{Spin}_{n}\right) \mid h^{0}(C, A(V) \otimes \kappa)>0\right\}
$$

are elements of the linear system $\mathbb{P} H^{0}\left(\mathcal{M}^{+}\left(\operatorname{Spin}_{n}\right), \mathcal{P}\right)$. Here $\mathcal{P}$ is a Pfaffian square-root of $\Theta(V)$,i.e., $\mathcal{P}^{2}=\Theta(V)$. To avoid existence problems, we work over the moduli stack. Then we shall prove

8.2. Proposition. A basis of the linear system $|\mathcal{P}|$ is given by the divisors $D_{\kappa}^{(n)}$ with $\kappa \in \vartheta(C)$, if $n$ is even, and $\kappa \in \vartheta(C)$ with $\epsilon(\kappa)=1$, if $n$ is odd. 
Proof. It is enough to prove linear independence, which is done by induction on $n$. For the first cases we refer to [B2] proposition A.8 $(n=2)$, theorem $1.2(n=3)$, proposition A.5 $(n=$ 4). The statement for $n=5$ will follow by pulling back $D_{\kappa}^{(5)} \subset \mathcal{M}^{+}\left(\operatorname{Spin}_{5}\right)$ under the map $\mathcal{M}^{+}\left(\operatorname{Spin}_{3}\right) \times \mathcal{M}^{+}\left(\operatorname{Spin}_{2}\right) \stackrel{\iota}{\longrightarrow} \mathcal{M}^{+}\left(\operatorname{Spin}_{5}\right)$. We observe that $\iota^{*}\left(D_{\kappa}^{(5)}\right)=p_{1}^{*}\left(D_{\kappa}^{(3)}\right)+p_{2}^{*}\left(D_{\kappa}^{(2)}\right)$. Since the $D_{\kappa}^{(3)}$ form a basis, we can conclude. Let us assume that the $D_{\kappa}^{(n)}$ 's are independent. Then the equality $\iota^{*}\left(D_{\kappa}^{(n+4)}\right)=p_{1}^{*}\left(D_{\kappa}^{(n)}\right)+p_{2}^{*}\left(D_{\kappa}^{(4)}\right)$ implies that the $D_{\kappa}^{(n+4)}$ 's are also independent.

3. The "twisted" Verlinde formula given in O2 conjecture 1.1, which computes the dimension of $H^{0}\left(\mathcal{M}^{-}\left(\operatorname{Spin}_{n}\right), \Theta(V)\right)$, can be deduced from a forthcoming work by Y. Laszlo and C. Sorger. Following the techniques of the paper [LS], the authors show that the "twisted" Verlinde space can be identified to a conformal block, whose dimension (worked out by C. Woodward) is given by Oxbury's formula.

4. We can prove a refinement of theorem 1.1 by considering the linear action of the Heisenberg group Heis on $H:=H^{0}\left(\mathcal{M}^{ \pm}\left(\operatorname{Spin}_{n}\right), \Theta(V)\right)$. By induction, we check that $H$ is a Heis-module of level 4 for the $J C[2]$-action described in (3.2). Hence the linear action of Heis factors through $J C[2]$ and we can consider its character space decomposition $H=\sum_{\eta \in J C[2]} H_{\eta}$. One proves that the image of $s_{\eta}^{ \pm}$is contained in $H_{\eta}$. Since $\sum_{\eta} s_{\eta}$ is surjective, we get equality $\operatorname{im}\left(s_{\eta}\right)=H_{\eta}$. So, we have isomorphisms, e.g. for $n$ odd,

$$
\forall \eta \in J C[2] \quad s_{\eta}^{ \pm}: H^{0}\left(P_{\eta}^{e v},(2 m+1) \Xi_{\eta}\right)_{ \pm}^{\vee} \stackrel{\sim}{\longrightarrow} H^{0}\left(\mathcal{M}^{ \pm}\left(\operatorname{Spin}_{2 m+1}\right), \Theta\left(\mathbb{C}^{2 m+1}\right)\right)_{\eta}
$$

\section{References}

[B1] A. Beauville: Fibrés de rang 2 sur une courbe, fibrés déterminant et fonctions thêta, Bull. Soc. Math. France 116 (1988), 431-448

[B2] A. Beauville: Fibrés de rang 2 sur une courbe, fibrés déterminant et fonctions thêta II, Bull. Soc. Math. France 119 (1991), 259-291

[B3] A. Beauville: Vector bundles on curves and generalized theta functions: recent results and open problems, Current topics in complex algebraic geometry (Berkeley, CA, 1992/93), Math. Sci. Res. Inst. Publ. 28, 17-33

[BNR] A. Beauville, M.S. Narasimhan, S. Ramanan: Spectral curves and the generalized theta divisor, J. reine angew. Math. 398 (1989), 169-179

[D1] R. Donagi: Non-Jacobians in the Schottky loci, Ann. of Math. 126 (1987), 193-217

[D2] R. Donagi: Big Schottky, Invent. Math. 89 (1987), 569-599

[DT] R. Donagi, L. Tu: Theta functions for SL(n) versus GL(n), Math. Res. Lett. 1, no.3 (1994), 345-357

[vGP] B. van Geemen, E. Previato: Prym varieties and the Verlinde formula, Math. Ann. 294 (1992), 741-754

[K] G. Kempf: Multiplication over abelian varieties, Amer. J. Math. 110 (1988), 765-773

[Kh] A. Khaled: Equations des variétés de Kummer, Math. Ann. 295 (1993), 685-701 
[M1] D. Mumford: Prym varieties I, In Contributions to Analysis, Academic Press, New York (1974)

[M2] D. Mumford: Theta-characteristics of an algebraic curve, Ann. Sci. Ec. Norm. Sup. 4 (1971), 181-192

[M3] D. Mumford: On the equations defining abelian varieties, Invent. Math. 1 (1966), 287-354

[LS] Y. Laszlo, C. Sorger: The line bundle on the moduli of parabolic G-bundles over curves and their sections, Ann. Sci. Ec. Norm. Sup. 30 (1997), 499-525

[O1] W.M. Oxbury: Prym varieties and the moduli of spin bundles, Algebraic geometry, ed. P.E. Newstead, Lect. Notes in Pure App. Math. vol. 200 (Marcel Dekker 1998), 351-376

[O2] W.M. Oxbury: Spin Verlinde spaces and Prym theta functions, Proc. London Math. Soc. (3) 78 (1999), 52-76

[OP] W.M. Oxbury, C. Pauly: $S U(2)$-Verlinde spaces as theta spaces on Pryms, Int. J. Math. 7 (3) (1996), 393-410

[OW] W.M. Oxbury, S.M.J. Wilson: Reciprocity laws in the Verlinde formulae for the classical groups, Trans. Amer. Math. Soc. 348 (1996), 2689-2710

[P] C. Pauly: On Pryms, rank 2 bundles and nonabelian theta functions, Int. J. Math. 8 (2) (1997), 267-287

[R1] S. Ramanan: Orthogonal and spin bundles over hyperelliptic curves, in Geometry and Analysis, papers dedicated to V.K. Patodi, Springer 1981

[R2] S. Ramanan: The Schottky configuration, preprint 1995

[S] C. Sorger: La formule de Verlinde, Séminaire Bourbaki, Vol. 1994/5, Astérisque 237 (1996), Exp. No. 794, 3, 87-114

C. Pauly

Laboratoire J.-A. Dieudonné

Université de Nice Sophia Antipolis

Parc Valrose

06108 Nice Cedex 02

France

email: pauly@math.unice.fr

S. Ramanan

Tata Institute of Fundamental Research

Homi Bhabha Road

Bombay 400005

India

email: ramanan@math.tifr.res.in 